\title{
COMORBILIDAD DE TUBERCULOSIS Y DIABETES: PROBLEMA AÚN SIN RESOLVER
}

\author{
César Ugarte-Gil1,2,a,b, David AJ Moore ${ }^{1,3, b, c}$
}

\begin{abstract}
RESUMEN
La comorbilidad entre tuberculosis y diabetes ha sido descrita desde principios del siglo XX. En países desarrollados donde se ha pasado de la disminución de la enfermedades infecciosas a un incremento de enfermedades no transmisibles, así como en aquellos países que aún tienen alta prevalencia de enfermedades infecciosas pero con incremento de las no transmisibles, se observa que la prevalencia de la comorbilidad entre tuberculosis y diabetes se está incrementando, volviendo el manejo clínico y el control a nivel de salud pública un nuevo reto para cualquier sistema de salud. Esta revisión intenta mostrar las evidencias disponibles hasta la actualidad y poner en conocimiento algunas líneas de investigación que se están comenzando a desarrollar para entender dicho problema. En países como el Perú donde hay una transición epidemiológica, mayor investigación permitiría conocer y describir mejor las características y el impacto de esta comorbilidad.
\end{abstract}

Palabras clave: Tuberculosis; Diabetes mellitus; Comorbilidad (fuente: DeCS BIREME).

\section{TUBERCULOSIS AND DIABETES CO-MORBIDITY: AN UNRESOLVED PROBLEM}

\begin{abstract}
Co-morbidity between tuberculosis and diabetes has been described since the early 20th century. In developed countries, where there has been a decrease of infectious diseases with an increase of non-communicable diseases, as well as those countries who still have a high prevalence of infectious diseases but an increase of non-communicable diseases, it is observed that the prevalence of co-morbidity between tuberculosis and diabetes is increasing, making clinical management and control at the public health level a new challenge for health systems. This review aims to show the current available evidence that can inform research lines being developed to understand the problem. In countries like Peru, where there is an epidemiological transition, further research could allow us to understand and describe in a better way the characteristics and impact of this co-morbidity.
\end{abstract}

Key words: Tuberculosis; Diabetes mellitus; Comorbidity (source: MeSH NLM).

\section{INTRODUCCIÓN}

La tuberculosis (TB), junto con la infección por el VIH, siguen siendo una de las enfermedades infecciosas más prevalentes en el mundo, con 8,6 millones de nuevos casos en 2012 y 1,3 millones de muertes ${ }^{(1)}$. La TB se denomina comúnmente como una "enfermedad de la pobreza" (2) y tiene impacto social significativo, en particular entre los segmentos económicamente activos de la población (PEA) ${ }^{(3)}$.

Se ha avanzado en varios objetivos dentro del Plan Mundial para Detener la TB 2011-2015 ${ }^{(4)}$, tales como la disminución de la incidencia mundial de la TB en los últimos dos años ${ }^{(1)}$. Sin embargo, el surgimiento de complicaciones como la TB multidrogorresistente (TB-MDR) la TB extremadamente resistente a drogas (TB-XDR), y comorbilidades como el VIH/SIDA y la diabetes mellitus (DM) ponen en riesgo el objetivo final de la eliminación mundial de TB para el año 2050 (4).

En las últimas décadas existe un incremento en la prevalencia no solo de las enfermedades transmisibles, sino también en las enfermedades no transmisibles (ENT), dando lugar a una doble carga en los países de bajos y medianos ingresos ${ }^{(5)}$. En el 2011, se estimaba que

\footnotetext{
Universidad Peruana Cayetano Heredia. Lima, Perú.

Johns Hopkins Bloomberg School of Public Health. Baltimore, EE. UU.

London School of Hygiene and Tropical Medicine. Londres, Reino Unido.

Médico cirujano; ${ }^{\mathrm{b}}$ master en Epidemiología; ${ }^{\mathrm{c}}$ médico infectólogo

Recibido: 23-12-13 Aprobado: 12-02-14
} 
había 366 millones de personas que vivían con DM en todo el mundo. Se proyecta que aumente a 552 millones para el año 2030 , con un incremento de la prevalencia en países en desarrollo ${ }^{(6)}$. América Latina está pasando por esta transición, con notables incrementos en la prevalencia de la hipertensión arterial, la diabetes y la hipertrigliceridemia, alcanzando niveles comparables a los EE. UU. y otros países desarrollados ${ }^{(7)}$. En el caso de la DM en concreto, la prevalencia en América Latina es de aproximadamente $5 \%$, en comparación con una estimación global del $8,3 \%{ }^{\left({ }^{(6)} \text {. }\right.}$

No existe suficientes datos epidemiológicos sobre la DM en el Perú: algunos estudios proporcionan estimaciones de prevalencia de DM alrededor de 5 a $7 \%{ }^{(7,8-11)}$. A diferencia del programa de TB en el Perú, que ofrece tratamiento y atención clínica de forma gratuita, no existe un programa similar para DM, al igual que con otras condiciones como son hipertensión, depresión o artritis. Considerando que un $56 \%$ de la población tiene que pagar de forma privada su servicios de salud (12), el manejo de la DM y otras enfermedades crónicas no transmisibles se hace difícil en el contexto del sistema de salud peruano.

Con relación a la evidencia disponible en relación de la comorbilidad TB-DM, esta es aún insuficiente, faltan estudios que evalúen el patrón fisiopatológico de la comorbilidad, factores genéticos yestudios farmacológicos. En el caso del Perú, la evidencia sobre el efecto de la DM y la TB es escasa, existen dos estudios observacionales realizados en pacientes hospitalizados y pacientes con alto riesgo de resistencia a los medicamentos anti-TB en los últimos 10 años ${ }^{(13,14)}$. Sin embargo, el nivel de evidencia no es suficiente, ya que no evalúa a la población general de pacientes con TB en el Perú.

\section{ASPECTOS EPIDEMIOLÓGICOS}

La asociación entre la TB y DM comenzó a recibir atención y ser descrita a mitad del siglo $X X{ }^{(15,16)}$, sin embargo, aún no se comprende totalmente. Uno de los conceptos clave que requieren investigación es la dirección de esta asociación, a pesar de que algunos estudios proveen la hipótesis de que la TB incrementa las posibilidades de que los pacientes desarrollen DM (debido al estado de hiperglicemia como resultado de la infección y por resultado del tratamiento TB) ${ }^{(17,18)}$, la mayoría de evidencia apoya la hipótesis de que la DM aumenta la susceptibilidad a desarrollar TB (debido a la inmunodeficiencia por presentar DM) ${ }^{(19)}$.

No existen muchos estudios que muestren las características de la población peruana y latinoamericana en relación con enfermedades crónicas como DM; un estudio comparó factores de riesgo cardiovascular entre la población de EE. UU. y la población de América Latina, encontrándose que la prevalencia de DM, obesidad, hipercolesterolemia e hipertrigliceridemia es mayor en los EE. UU. comparado con América Latina ${ }^{(7)}$. Al proyectarse que el $80 \%$ de los pacientes con DM vivirán en los países en desarrollo para el año $2030{ }^{(6)}$, es importante conocer las características de estos pacientes y los factores de riesgo para hacer frente a las intervenciones adecuadas para prevenir la aparición de DM.

En el caso de la asociación TB-DM en el Perú, también hay falta de información. Los dos estudios arriba mencionados incluyen pacientes de grupos específicos, uno de los estudios fue una serie de casos de pacientes hospitalizados en Lima ${ }^{(13)}$ y el otro es un estudio de cohorte en pacientes con alto riesgo para TB resistente, encontrándose que un $11 \%$ tenía DM ${ }^{(14)}$. Sin embargo, como los mismo autores refieren, ha habido limitaciones en el diseño del estudio que podrían llevar a un sesgo de selección, por lo que la prevalencia de DM en la población de TB en el Perú necesita ser evaluada.

Uno de los problemas es el tamizaje para DM a los pacientes con TB, y viceversa, debido a la variabilidad que ofrecen los métodos diagnósticos disponibles. Una revisión sistemática que evalúa el tamizaje bidireccional muestra que hay una alta prevalencia (pero con rangos amplios de más de 30 puntos porcentuales) de TB entre los pacientes de DM y alta prevalencia de DM entre los pacientes con TB ${ }^{(20)}$. La sensibilidad de las pruebas para DM (como HbA1c o hemoglobina glicosilada, glucosa en ayunas, glucosa al azar, tolerancia oral a la glucosa entre otros) es variable, por lo que podría haber un registro no completo de los casos TB-DM. La sensibilidad de la $\mathrm{HbA} 1 \mathrm{c}$ puede ir desde $65 \%$ hasta $88 \%$ dependiendo del punto de corte $(6,5 \circ 6,1 \%)^{(21)}$ y se ha visto que detecta más que la glucosa al azar (9), sin embargo, debido a su costo es de difícil acceso. La Norma Técnica de Tuberculosis en el Perú indica que el tamizaje debe hacerse a toda persona con diagnóstico de TB con prueba de glicemia en ayunas ${ }^{(22)}$, prueba que es de mas fácil acceso y, en este caso, es cubierta por el programa nacional, pero que no es de fácil cumplimiento debido a que los pacientes tienen que ir en ayunas para la toma de muestra.

\section{ASPECTOS FISIOPATOLÓGICOS}

La respuesta inmune en las personas con DM es compleja y aún no se entiende por completo. Algunos estudios muestran que un aumento en el tejido 
adiposo (como el que se da en DM) esta asociado con un incremento de la producción de citoquinas proinflamatorias (tales como TNF- $\alpha$, IL-6, IL-8, IL-12) (23-27). Estudios en ratones con TB-DM mostraron un aumento de citoquinas proinflamatorias (TNF- $\alpha$, IFN-y e IL-1b) comparados con ratones con TB y no DM ${ }^{(28,29)}$. En un estudio con pacientes con TB, TB-DM y controles, se encontró que hay un incremento de los niveles de TNF- $\alpha$ y IFN-y en los pacientes con TB-DM comparado con los pacientes con solo TB (30). La evidencia adicional sobre la relación de la hiperglucemia y el riesgo de infección ${ }^{(31,32)}$ podría explicar la mayor gravedad en la presentación clínica en los pacientes TB-DM en comparación con los pacientes con TB y no $D M{ }^{(19)}$. Considerando que la respuesta inmune en los pacientes con TB aún no esta entendida por completo, se hace necesario más estudios inmunológicos y genéticos para poder entender la interacción entre estas dos enfermedades.

\section{ASPECTOS CLÍNICOS}

El impacto de la DM en la TB ha recibido recientemente más atención, como es evidente a través de un aumento de las publicaciones sobre el tema en los últimos años. Ha habido varias revisiones narrativas ${ }^{(15,19,33,34)}$ y hay solo dos metaanálisis de la evaluación de esta asociación ${ }^{(35,36)}$. En general, el cuadro clínico de un paciente con TB-DM tiende a ser más severo por factores de inmunosupresión, diferente farmacocinética de los medicamentos para TB y DM y comorbilidades que afectan el cuadro clínico en general como hipertensión u obesidad. Existe un incremento en el riesgo de TB activa en los pacientes con DM (RR: 3,11; IC 95\% 2,27-4,26) en estudios de tipo cohorte ${ }^{(36)}$. Asimismo, el riesgo de muerte en los pacientes con TB-DM es mayor comparado con los pacientes con solo TB (RR: 1,89; IC 95\%: 1,52-2,36) y el riesgo de recaída es mayor entre los pacientes con TB-DM comparado con los pacientes TB no DM (RR: 3,89; IC 95\%: 2,43-6,23) ${ }^{(35)}$. A pesar de que una de las limitaciones de ambos metaanálisis es que los estudios incluidos son en su mayoría desarrollados en países en desarrollo, hay nueva evidencia en países en desarrollo que confirmaría la evidencia de la asociación TB-DM con un peor cuadro clínico en los pacientes con TB-DM ${ }^{(37-40)}$

Una de las formas de medir si el tratamiento para TB esta siendo exitoso o no, es el resultado negativo para $M$. tuberculosis en esputo al segundo mes de tratamiento, a falta de mejores marcadores de éxito durante el tratamiento (41). Estudios sugerirían que en los pacientes con TB-DM hay una menor tasa de conversión de del resultado de esputo de positivo a negativo comparado con pacientes con TB no DM ${ }^{(38,40)}$, sin embargo, también existen estudios que mostrarían que no habría diferencia entre pacientes TB-DM y TB no DM ${ }^{(37)}$, haciéndose necesario realizar nuevos estudios en esta área.

Uno de los retos que se da en los pacientes con TBDM es el manejo terapéutico, se ha demostrado que los niveles de las drogas anti-TB en plasma están debajo de los valores terapéuticos en pacientes con TB-DM (42-44). Esto, añadido a la asociación entre hiperglicemia y el uso de drogas anti-TB, hace que el manejo terapéutico sea difícil. Aún no hay estudios aleatorizados que evalúen un tratamiento adecuado para TB-DM, y tampoco hay suficiente evidencia que evalúe el efecto del uso de insulina o metformina en este tipo de pacientes ${ }^{(45)}$, siendo el tratamiento recomendado en el Perú el uso de insulina en pacientes con TB-DM ${ }^{(22)}$.

\section{INVESTIGACIONES FUTURAS}

En los últimos años se están produciendo más evidencia en relación con esta comorbilidad TB-DM, con un incremento especialmente de publicaciones en países en desarrollo. En el Perú se esta desarrollando el proyecto TANDEM (http://tandemfp7.eu), gracias al cual se estudiará el efecto del tamizaje bidireccional TB-DM, asimismo, se evaluará la prevalencia de DM en los pacientes con TB en población peruana. Este proyecto multicéntrico (cuyo enrolamiento de participantes se realizarán en Perú, Sudáfrica, Indonesia y Rumania, con la colaboración de instituciones de Inglaterra, Holanda, Alemania y Nueva Zelandia) donde se evaluara dichos objetivos, además de poder contribuir con datos para investigaciones clínicas (mortalidad, tiempo de negativización de esputo, severidad de presentación clínica), inmunológicas, genéticas y farmacocinéticas en el futuro. Algunas preguntas que aún no han sido claramente contestadas y que no hay evidencia clara aún son:

- ¿Cuál es el método más sensible y costo-efectivo para diagnosticar DM en los pacientes con TB y viceversa?

- ¿Cuál es régimen terapéutico adecuado para manejar DM en los pacientes con TB?

- ¿Cuál es el efecto de las drogas anti-TB en el manejo de DM?

- ¿Cual es el riesgo de recaída de TB en los pacientes con comorbilidad TB-DM?

- ¿Cuáles son los factores asociados a la comorbilidad TB-DM en la población peruana? 
- ¿Una mejor vigilancia o control de glicemia mejora el resultado de tratamiento de TB y/o DM en los pacientes con TB-DM?

\section{CONCLUSIONES}

En momentos en que hay una transición epidemiológica en países como el Perú, se hace necesaria mayor investigación de dos enfermedades como TB y DM en nuestra población. Hay varios vacíos en la evidencia, no solo en la parte clínica y en ciencias básicas, sino también en factores epidemiológicos para poder describir el impacto y las características de los pacientes TB-DM a nivel local, sugiriéndose que sea monitoreada a nivel del Programa Nacional de TB para su evaluación anual (como se hace con la coinfección TB/VIH). Habiéndose encontrado que hay un incremento de riesgo en el aumento de TB activa en los pacientes con DM, el control de la TB, sin un adecuado manejo a nivel poblacional de DM (así como otras comorbilidades como VIH/SIDA, depresión o enfermedad renal), seria infructuoso, con el consecuente impacto en todo el sistema de salud.

Conflictos de Interés: los autores no reportan conflictos de interés.

Fuentes de financiamiento: CUG y DAJM son apoyados por el proyecto TANDEM, financiado por el European Community's Seventh Framework Programme (FP7/20072013) bajo el acuerdo 305279. CUG tiene apoyo del proyecto Peru ICOHRTA Network for AIDS/TB Research Training (NIH Grant 1U2RTW007368-01A1- Fogarty International Center, Lima Perú)

\section{REFERENCIAS BIBLIOGRÁFICAS}

1. World Health Organization (WHO). Global Tuberculosis Report 2013. Ginebra: WHO; 2013.

2. Lonnroth K, Jaramillo E, Williams BG, Dye C, Raviglione M. Drivers of tuberculosis epidemics: the role of risk factors and social determinants. Soc Sci Med. 2009;68(12):2240-6. doi: 10.1016/j. socscimed.2009.03.041.

3. Rajeswari R, Balasubramanian R, Muniyandi M, Geetharamani S, Thresa X, Venkatesan P. Socioeconomic impact of tuberculosis on patients and family in India. Int J Tuberc Lung Dis. 1999;3(10):86977.

4. World Health Organization (WHO). The global plan to stop TB 2011-2015 [Internet]. Ginebra: WHO; 2011 [citado el 20 de diciembre de 2013]. Disponible en: http://www.stoptb.org/assets/ documents/global/plan/TB GlobalPlanToStopTB2011-2015.pdf

5. Lim SS, Vos T, Flaxman AD, Danaei G, Shibuya K, AdairRohani $\mathrm{H}$, et al. A comparative risk assessment of burden of disease and injury attributable to 67 risk factors and risk factor clusters in 21 regions, 1990-2010: a systematic analysis for the Global Burden of Disease Study 2010. Lancet. 2012;380(9859):2224-60. doi: 10.1016/S0140-6736(12)61766-8.

6. International Diabetes Federation. IDF Diabetes Atlas 2011 [Internet]. Brussels: International Diabetes Federation; c2013 [citado el 20 de diciembre de 2013]. Disponible en: http://www.eatlas.idf.org

7. Miranda JJ, Herrera VM, Chirinos JA, Gómez LF, Perel P, Pichardo R, et al. Major cardiovascular risk factors in Latin America: a comparison with the United States. The Latin American Consortium of Studies in Obesity (LASO). PLoS One. 2013;8(1):e54056. doi: 10.1371/ journal.pone.0054056.

8. Garcia F, Solis J, Calderon J, Luque E, Neyra L, Manrique $\mathrm{H}$, et al. Prevalencia de diabetes mellitus y factores de riesgo relacionados en una población urbana. Rev Soc Peru Med Interna. 2007;20(3):90-4.

9. Miranda JJ, Bernabe-Ortiz A, Stanojevic S, Malaga G, Gilman RH, Smeeth L. A1C as a diagnostic criteria for diabetes in low- and middleincome settings: evidence from Peru. PLoS One. 2011;6(3):e18069. doi: 10.1371/journal.pone.0018069.

10. Miranda JJ, Gilman RH, Smeeth L. Differences in cardiovascular risk factors in rural, urban and rural- to-urban migrants in Peru. Heart. 2011;97(10):787-96. doi: 10.1136/ hrt.2010.218537.

11. Zaman MJ, Loret de Mola C, Gilman RH, Smeeth L, Miranda JJ. The prevalence of angina symptoms and association with cardiovascular risk factors, among rural, urban and rural to urban migrant populations in Peru. BMC Cardiovasc Disord. 2010;10:50. doi: 10.1186/14712261-10-50.

12. Loh LC, Ugarte-Gil C, Darko K. Private sector contributions and their effect on physician emigration in the developing world. Bull World Health Organ. 2013;91(3):227-33. doi: 10.2471/BLT.12.110791.

13. Delgado Rospigliosi JL, Seclen Santisteban SN, Gotuzzo Herencia E. Tuberculosis en pacientes con diabetes mellitus: Un estudio epidemiológico y clínico en el Hospital Nacional Cayetano Heredia. Rev Med Hered. 2006;17(3):132-40.

14. Magee MJ, Bloss E, Shin SS, Contreras C, Huaman HA, Ticona JC, et al. Clinical characteristics, drug resistance, and treatment outcomes among tuberculosis patients with diabetes in Peru. Int J Infect Dis. 2013;17(6):e404-12. doi: 10.1016/j.ijid.2012.12.029. 
15. Banyai AL.Diabetes and tuberculosis. Dis Chest 1959;36:238-42.

16. Cooper DA, Boucot KR, Dillon ES, Meier P, Richardson R. Tuberculosis among diabetics: the Philadelphia survey. Trans Annu Meet Natl Tuberc Assoc. 1951;47:175-81.

17. Basoglu OK, Bacakoglu F, Cok G, Sayiner A, Ates M. The oral glucose tolerance test in patients with respiratory infections. Monaldi Arch Chest Dis. 1999;54(4):307-10.

18. Oluboyo PO, Erasmus RT. The significance of glucose intolerance in pulmonary tuberculosis. Tubercle. $1990 ; 71(2): 135-8$

19. Dooley KE, Chaisson RE. Tuberculosis and diabetes mellitus: convergence of two epidemics. Lancet Infect Dis. 2009;9(12):73746.

20. Jeon CY, Harries AD, Baker MA, Hart JE, Kapur A, Lönnroth K, et al. Bi-directional screening for tuberculosis and diabetes: a systematic review. Trop Med Int Health. 2010;15(11):1300-14. doi: 10.1111/j.1365-3156.2010.02632.x.

21. Kumar PR, Bhansali A, Ravikiran M, Bhansali S, Dutta P, Thakur JS, et al. Utility of glycated hemoglobin in diagnosing type 2 diabetes mellitus: a community-based study. J Clin Endocrinol Metab. 2010;95(6):2832-5. doi: $10.1210 /$ jc.2009-2433.

22. Perú, Ministerio de Salud (MINSA). Norma Técnica de Salud para la atención integral de las personas afectadas por Tuberculosis. Resolución Ministerial $N^{\circ} 715$ 2013/MINSA del 8 de noviembre del 2013. Lima: MINSA; 2013

23. Guzman-Flores JM, Lopez-Briones S. Células de la inmunidad innata y adaptativa en la diabetes mellitus tipo 2 y obesidad. Gac Med Mex. 2012;148:381-9.

24. Koh GC, Peacock SJ, van der Poll T, Wiersinga WJ. The impact of diabetes on the pathogenesis of sepsis. Eur J Clin Microbiol Infect Dis. 2012;31(4):379-88. doi: 10.1007/s10096-011-1337-4..

25. Rodewald HR, Feyerabend TB. Widespread immunological functions of mast cells: fact or fiction? Immunity. 2012;37(1):13-24. doi: 10.1016/j.immuni.2012.07.007.
26. Sell H, Habich C, Eckel J. Adaptive immunity in obesity and insulin resistance. Nat Rev Endocrinol. 2012;8(12):709-16. doi: 10.1038/ nrendo.2012.114..

27. Sun S, Ji Y, Kersten S, Qi L. Mechanisms of inflammatory responses in obese adipose tissue. Annu Rev Nutr. 2012;32:26186. doi: 10.1146/annurevnutr-071811-150623.

28. Martens GW, Arikan MC, Lee J, Ren F, Greiner D, Kornfeld H. Tuberculosis susceptibility of diabetic mice. Am J Respir Cell Mol Biol. 2007;37(5):518-24.

29. Vallerskog T, Martens GW, Kornfeld H. Diabetic mice display a delayed adaptive immune response to Mycobacterium tuberculosis. J Immunol. 2010;184(11):6275-82. doi: 10.4049/jimmunol.1000304.

30. Zheng Y, Ma A, Wang Q, Han X, Cai J, Schouten EG, et al. Relation of leptin, ghrelin and inflammatory cytokines with body mass index in pulmonary tuberculosis patients with and without type 2 diabetes mellitus. PLoS One. 2013;8(11):e80122. doi: 10.1371/journal.pone.0080122.

31. King JT Jr, Goulet JL, Perkal MF, Rosenthal RA. Glycemic control and infections in patients with diabetes undergoing noncardiac surgery. Ann Surg. 2011 Jan;253(1):158-65. doi: 10.1097/SLA.0b013e3181f9bb3a.

32. Rayfield EJ, Ault MJ, Keusch GT, Brothers MJ, Nechemias C, Smith $\mathrm{H}$. Infection and diabetes: the case for glucose control. Am J Med. 1982;72(3):439-50

33. Bailey SL, Grant P. 'The tubercular diabetic': the impact of diabetes mellitus on tuberculosis and its threat to global tuberculosis control. Clin Med. 2011;11(4):344-7.

34. Kapur A, Harries AD. The double burden of diabetes and tuberculosis Public health implications. Diabetes Res Clin Pract. 2013 Jan 7. pii: S0168-8227(12)00497-4. doi: 10.1016/j.diabres.2012.12.001. [Epub ahead of print]

35. Baker MA, Harries AD, Jeon CY, Hart JE, Kapur A, Lönnroth K, et al. The impact of diabetes on tuberculosis treatment outcomes: a systematic review. BMC Med. 2011;9:81. doi: 10.1186/1741-
7015-9-81.

36. Jeon CY, Murray MB. Diabetes mellitus increases the risk of active tuberculosis: a systematic review of 13 observational studies. PLoS Med. 2008;5(7):e152. doi: 10.1371/ journal.pmed.0050152.

37. Faurholt-Jepsen D, Range N, PrayGod G, Jeremiah K, FaurholtJepsen M, Aabye MG, et al. Diabetes is a strong predictor of mortality during tuberculosis treatment: a prospective cohort study among tuberculosis patients from Mwanza, Tanzania. Trop Med Int Health. 2013;18(7):822-9. doi: 10.1111/ tmi. 12120 .

38. Jiménez-Corona ME, Cruz-Hervert LP, García-García L, Ferreyra-Reyes L, Delgado-Sánchez G, BobadillaDel-Valle $\mathrm{M}$, et al. Association of diabetes and tuberculosis: impact on treatment and posttreatment outcomes. Thorax. 2013;68(3):214-20. doi: 10.1136/ thoraxjnl-2012-201756.

39. Mi F, Tan S, Liang L, Harries AD, Hinderaker SG, Lin Y, et al. Diabetes mellitus and tuberculosis: pattern of tuberculosis, two-month smear conversion and treatment outcomes in Guangzhou, China. Trop Med Int Health. 2013 Sep 23. doi: 10.1111/ tmi.12198. [Epub ahead of print]

40. Reis-Santos B, Locatelli R, Horta BL, Faerstein E, Sanchez MN, Riley LW, et al. Socio-demographic and clinical differences in subjects with tuberculosis with and without diabetes mellitus in Brazil--a multivariate analysis. PLoS One. 2013;8(4):e62604. doi: 10.1371/ journal.pone.0062604.

41. Horne DJ, Royce SE, Gooze L, Narita M, Hopewell PC, Nahid P, et al. Sputum monitoring during tuberculosis treatment for predicting outcome: systematic review and meta-analysis. Lancet Infect Dis. 2010;10(6):387-94. doi: 10.1016/ S1473-3099(10)70071-2.

42. Babalik A, Ulus IH, Bakirci N, Kuyucu T, Arpag H, Dagyildizi $\mathrm{L}$, et al. Plasma concentrations of isoniazid and rifampin are decreased in adult pulmonary tuberculosis patients with diabetes mellitus. Antimicrob Agents Chemother. 2013;57(11):5740-2. doi: 10.1128/ AAC.01345-13. 
43. Nijland HM, Ruslami R, Stalenhoef JE, Nelwan EJ, Alisjahbana B, Nelwan RH, et al. Exposure to rifampicin is strongly reduced in patients with tuberculosis and type 2 diabetes. Clin Infect Dis. 2006;43(7):848-54.

44. Ruslami R, Nijland HM, Adhiarta IG, Kariadi SH, Alisjahbana B, Aarnoutse RE, et al. Pharmacokinetics of antituberculosis drugs in pulmonary tuberculosis patients with type 2 diabetes. Antimicrob Agents Chemother. 2010;54(3):1068-74. doi: 10.1128/ AAC.00447-09.

45. Ruslami R, Aarnoutse RE, Alisjahbana B, van der Ven AJ, van Crevel R. Implications of the global increase of diabetes for tuberculosis control and patient care. Trop Med Int Health. 2010;15(11):1289-
$99 . \quad$ doi: $\quad 10.1111 /$ j.1365-

3156.2010.02625.x.

Correspondencia: César Ugarte Gil

Dirección: Universidad Peruana Cayetano Heredia. Av. Honorio Delgado 430 San Martin de Porres, Lima, Perú Teléfono: 51 - 1- 4823910

Correoelectrónico: cesar.ugarte@upch.pe

\section{Descarge de forma gratuita los contenidos de la}

Revista Peruana de Medicina Experimental y Salud Pública, ingrese a WWW.ins.gob.pe, seleccione el ícono de la revista y envíenos sus datos.

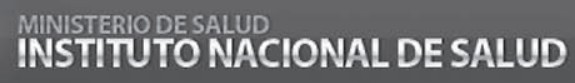

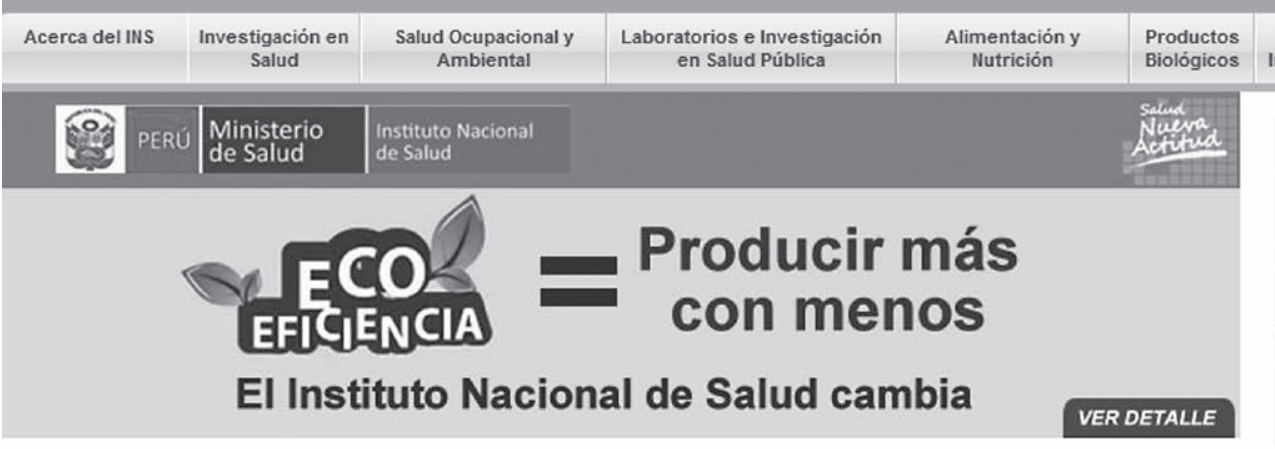

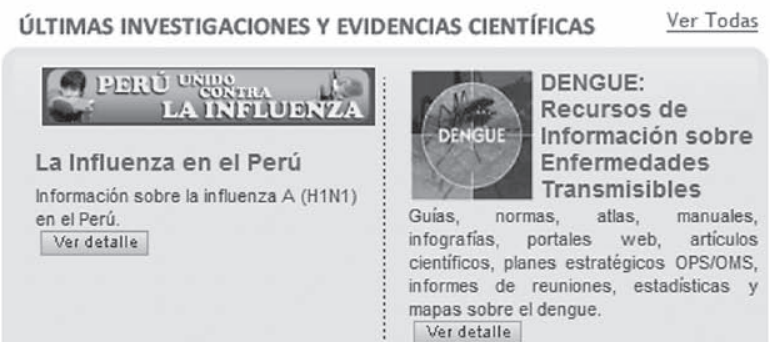

Ver detalle
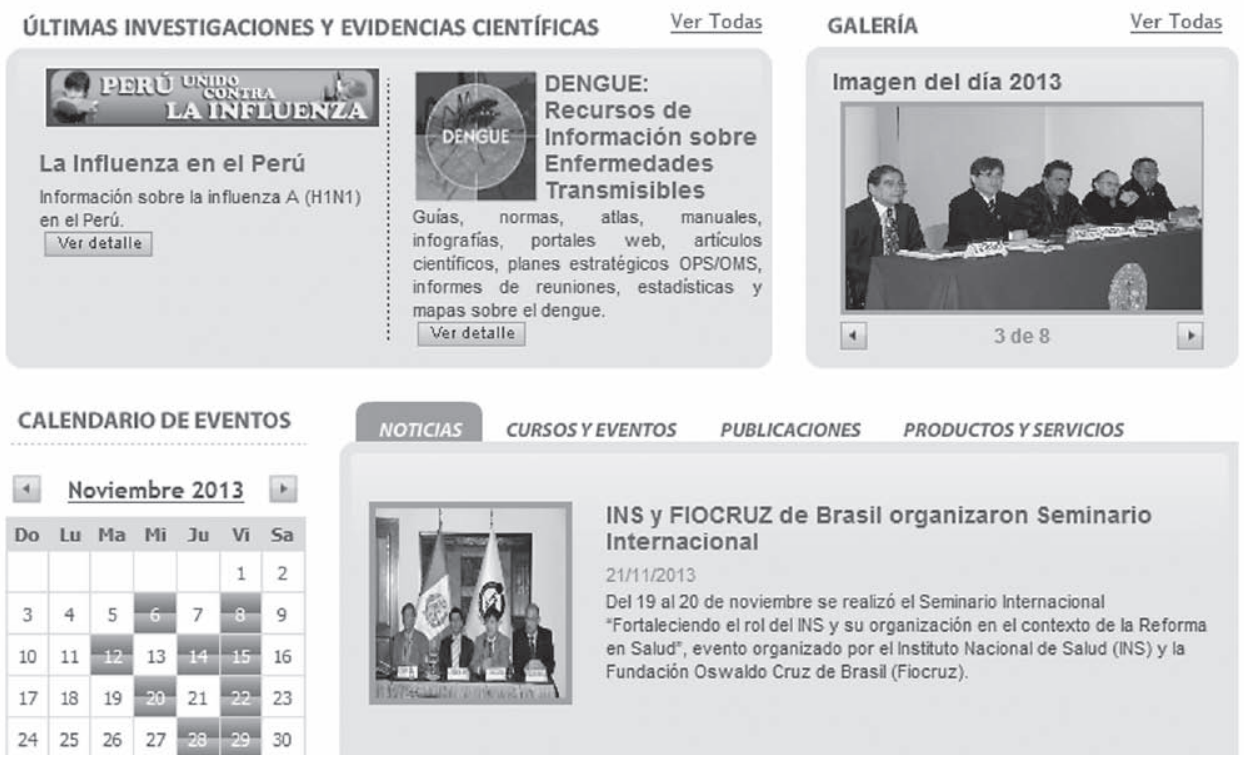

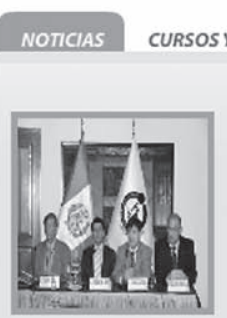

INS y FIOCRUZ de Brasil organizaron Seminario Internacional

21/11/2013

Del 19 al 20 de noviembre se realizó el Seminario Internacional

"Fortaleciendo el rol del INS y su organización en el contexto de la Reform

en Salud", evento organizado por el Instituto Nacional de Salud (INS) y la

Fundación Oswaldo Cruz de Brasil (Fiocruz).
Salud Control de Calidad Intercultural de Medicamentos

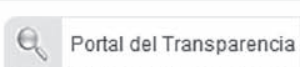

N Gestión de calidad

6 Directorio Telefónico

27 noviembre del 2013

Agregar a favoritos

- Enviar a un amigo

Enlaces de Interes

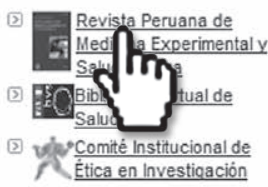

Yhe Ética en Investioación

$\underline{\text { Ver más }}$

Centro

INVESTIGACION

Investigación en Salud

Ir al home 